\title{
Long-Distance Properties of Landau Gluon and Ghost Propagators and Deconfinement
}

\author{
Attilio Cucchieri \\ Instituto de Física de São Carlos, Universidade de São Paulo, Caixa Postal 369, 13560-970 São \\ Carlos, SP, Brazil \\ E-mail: attilio@ifsc.usp.br \\ Tereza Mendes* \\ Instituto de Física de São Carlos, Universidade de São Paulo, Caixa Postal 369, 13560-970 São \\ Carlos, SP, Brazil \\ E-mail: mendes@ifsc.usp.br
}

\begin{abstract}
We investigate numerically the long-distance properties of gluon and ghost propagators in Landau gauge on the lattice, for the $\mathrm{SU}(2)$ case. By considering electric and magnetic gluon propagators at nonzero temperature, we extract Debye screening masses and look for signs of deconfinement around the critical temperature. Our results are related to the zero-temperature behavior of infrared propagators for comparison.
\end{abstract}

34th annual International Symposium on Lattice Field Theory

24-30 July 2016

University of Southampton, UK

${ }^{*}$ Speaker. 


\section{Landau-Gauge Propagators and Temperature}

Gluonic correlations of Yang-Mills theory at nonzero temperature are encoded in the chromoelectric (i.e. longitudinal) sector of the gluon propagator, which is a gauge-dependent quantity. At high temperatures, deconfinement should be felt in the electric gluon propagator as an exponential fall-off at long distances, defining a screening length and conversely a screening mass [1]. As for the magnetic (i.e. transverse) sector, the dimensional-reduction picture (based on the 3D-adjointHiggs model) suggests a confined magnetic gluon, associated to a nontrivial magnetic mass.

Lattice studies of gluon and ghost propagators in Landau gauge have shown that both the ghost propagator and the magnetic gluon propagator are essentially unaffected by temperature. The longitudinal propagator $D_{L}(p)$, on the other hand, shows significantly different behavior for different temperatures. As soon as a nonzero temperature is introduced in the system, $D_{L}(p)$ increases considerably. More precisely, for all fixed temperatures, the curve described by $D_{L}(p)$ seems to reach a plateau in the low-momentum region [2]. As the temperature is increased, this plateau increases slightly until, approaching the phase transition from below, it has been observed to rise further and then, just above the transition temperature, to drop sharply. This has been interpreted as a sign of singular behavior of the longitudinal gluon propagator around $T_{c}$ and, in fact, it has been related to several proposals of a new order parameter for the deconfinement transition (see e.g. [3]). Of course, a relevant question is, then, whether this singularity survives the inclusion of dynamical quarks in the theory $[4,5]$.

Let us mention that, at all investigated temperatures, the infrared plateau just described is not long enough to justify a fit to the Yukawa form

$$
D_{L}(p)=C \frac{1}{p^{2}+m^{2}}
$$

predicted at high temperatures. If this were the case, $D_{L}(0)^{-1 / 2}$ would provide a natural (temperature-dependent) mass scale. Note that this value depends also on the global constant $C$. On the other hand, the so-called Gribov-Stingl forms involve complex-conjugate poles, defining real and imaginary masses (independently of $C$ ).

\section{Electric Screening Masses}

In order to characterize a mass scale around $T_{c}$, we consider a generalized fitting form of the Gribov-Stingl type

$$
D_{L, T}(p)=C\left[\frac{1+d p^{2}}{\left(p^{2}+a\right)^{2}+b^{2}}\right]^{\eta} .
$$

This form allows for two (complex-conjugate) poles, with masses $m^{2}=a \pm i b$, where $m=$ $m_{R}+i m_{I}$. The mass $m$ thus depends only on $a, b$ and not on the normalization $C$. Note that, for given values of $a, b, d, \eta$, the global constant $C$ is fixed by the renormalization condition, so that there are only four free parameters in (2.1).

The parameter $\eta$ should be 1 if the fitting form also describes the large-momenta region (from our infrared data we get $\eta \neq 1$ ). Recall that at high temperatures one usually defines the electric 
screening mass as the scale determining the exponential decrease of the real-space propagator at large distances, which is equivalent to $D_{L}(0)^{-1 / 2}$ in the case of a real pole. We therefore expect to observe $m_{I} \rightarrow 0$ (i.e. $b \rightarrow 0$ ) for the longitudinal gluon propagator at high temperature.

Note that, if the propagator has the above form (and $\eta=1$ ), then the screening mass defined by $D_{L}(0)^{-1 / 2}=\sqrt{\left(a^{2}+b^{2}\right) / C}$ mixes the complex and imaginary masses $m_{R}$ and $m_{I}$ and depends on the (a priori arbitrary) normalization $C$.

We generally find good fits to the above form with nonzero real and imaginary parts of the pole masses in all cases. The form is well suited for the longitudinal and the transverse cases. For the transverse propagator $D_{T}(p)$, the masses $m_{R}$ and $m_{I}$ are of comparable size (around 0.6 and 0.4 $\mathrm{GeV}$ respectively). The same holds for $D_{L}(p)$, but in this case the relative size of the imaginary mass seems to decrease with increasing temperature.

The use of a Gribov-Stingl form is motivated by the behavior of the gluon propagator at $T=0$, where this type of expression has been shown to describe well the data in $3 d$ and $4 d$ [6]. (Note that the $3 d$ case may be considered as the $T \rightarrow \infty$ limit of the $4 d$ case.) Here, however, we need to incorporate the anomalous-dimension exponent $\eta$, corresponding to a nontrivial analytic structure of the gluon propagator involving branch cuts, as proposed in [7].

\section{Numerical Simulations}

We have considered the pure $\mathrm{SU}(2)$ case, with a standard Wilson action and lattice sizes $N_{s}^{3} \times N_{t}$ ranging from $48^{3} \times 4$ to $192^{3} \times 16$. For our runs we employ a cold start, performing a projection on positive-Polyakov-loop configurations. Also, gauge fixing is implemented using stochastic overrelaxation. The gluon dressing functions are normalized to 1 at $2 \mathrm{GeV}$. We considered several values of the lattice parameter $\beta$, allowing a broad range of temperatures. Our procedure for determining the physical temperature $T$ is described in [8]. The momentum-space longitudinal gluon propagator $D_{L}(p)$ is given by the scalar function

$$
D_{L}(p)=\frac{1}{N_{c}^{2}-1}\left\langle A_{0}^{a}(p) A_{0}^{a}(-p)\right\rangle
$$

where $N_{c}=2$, we take $p_{0}=0$ and $A_{\mu}(p)$ is the Fourier transform of the gauge field

$$
A_{\mu}^{a}(x) \lambda^{a}=\left.\frac{U_{\mu}(x)-U_{\mu}^{\dagger}(x)}{2 i}\right|_{\text {traceless }}
$$

satisfying the Landau gauge condition. 


\section{Results}
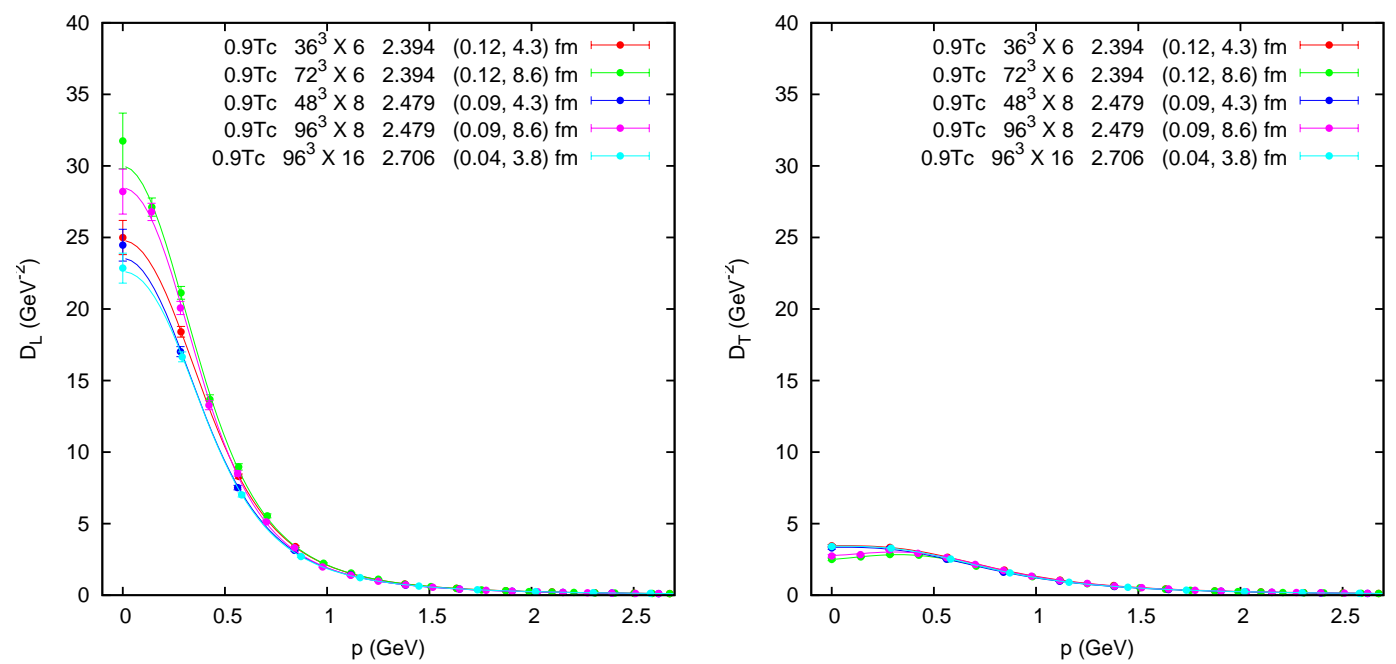

In the above plots we show the longitudinal (left) and transverse (right) gluon propagators $D_{L}$ and $D_{T}$ as functions of the (unimproved) lattice momentum $p$ together with the corresponding fitting functions. We plot data for $T / T_{c} \approx 0.9$ and several lattice sizes.

A table with the mass scales obtained from fits of $D_{L}$ and $D_{T}$ is shown below.

\begin{tabular}{|c|c|c|c|c|c|}
\hline$T / T_{c}$ & $N_{s}^{3} \times N_{t}$ & $m_{R}^{(E)}$ & $m_{I}^{(E)}$ & $m_{R}^{(M)}$ & $m_{I}^{(M)}$ \\
\hline 0 & $64^{3} \times 64$ & $0.69 \mathrm{GeV}$ & $0.34 \mathrm{GeV}$ & $0.77 \mathrm{GeV}$ & $0.42 \mathrm{GeV}$ \\
0.25 & $96^{3} \times 16$ & $0.82 \mathrm{GeV}$ & $0.33 \mathrm{GeV}$ & $0.64 \mathrm{GeV}$ & $0.29 \mathrm{GeV}$ \\
0.5 & $48^{3} \times 8$ & $0.69 \mathrm{GeV}$ & $0.21 \mathrm{GeV}$ & $0.67 \mathrm{GeV}$ & $0.44 \mathrm{GeV}$ \\
0.7 & $96^{3} \times 8$ & $0.58 \mathrm{GeV}$ & $0.23 \mathrm{GeV}$ & $0.58 \mathrm{GeV}$ & $0.25 \mathrm{GeV}$ \\
0.9 & $96^{3} \times 16$ & $0.53 \mathrm{GeV}$ & $0.24 \mathrm{GeV}$ & $0.69 \mathrm{GeV}$ & $0.42 \mathrm{GeV}$ \\
0.98 & $96^{3} \times 8$ & $0.49 \mathrm{GeV}$ & $0.22 \mathrm{GeV}$ & $0.65 \mathrm{GeV}$ & $0.44 \mathrm{GeV}$ \\
1.0 & $96^{3} \times 8$ & $0.56 \mathrm{GeV}$ & $0.19 \mathrm{GeV}$ & $0.58 \mathrm{GeV}$ & $0.31 \mathrm{GeV}$ \\
1.05 & $96^{3} \times 8$ & $0.70 \mathrm{GeV}$ & $0.26 \mathrm{GeV}$ & $0.58 \mathrm{GeV}$ & $0.36 \mathrm{GeV}$ \\
2.0 & $96^{3} \times 8$ & $1.08 \mathrm{GeV}$ & $0.15 \mathrm{GeV}$ & $0.83 \mathrm{GeV}$ & $0.35 \mathrm{GeV}$ \\
\hline
\end{tabular}

\section{References}

[1] D. J. Gross, R. D. Pisarski, L. G. Yaffe, Rev. Mod. Phys. 53, 43 (1981).

[2] A. Cucchieri, A. Maas and T. Mendes, Phys. Rev. D 75, 076003 (2007).

[3] P. J. Silva, O. Oliveira, P. Bicudo and N. Cardoso, Phys. Rev. D 89, 074503 (2014).

[4] V. G. Bornyakov and V. K. Mitrjushkin, Int. J. Mod. Phys. A 27, 1250050 (2012).

[5] R. Aouane, F. Burger, E. -M. Ilgenfritz, M. Muller-Preussker and A. Sternbeck, Phys. Rev. D 87, 114502 (2013).

[6] A. Cucchieri, D. Dudal, T. Mendes and N. Vandersickel, Phys. Rev. D 85, 094513 (2012).

[7] R. Alkofer, W. Detmold, C. S. Fischer and P. Maris, Phys. Rev. D 70, 014014 (2004).

[8] A. Cucchieri and T. Mendes, PoS FACESQCD, 007 (2010). 